\title{
Pathological and radiological changes in resected lung specimens in Mycobacterium avium intracellulare complex disease
}

\author{
J. Fujita*, Y. Ohtsuki ${ }^{\ddagger}$ I. Suemitsu**, E. Shigeto ${ }^{\star}$, I. Yamadori ${ }^{+}$, Y. Obayashi*, H. Miyawaki\#, \\ N. Dobashi*, T. Matsushima ${ }^{\$}$, J. Takahara*
}

Pathological and radiological changes in resected lung specimens in Mycobacterium avium intracellulare complex disease. J. Fujita, Y. Ohtsuki, I. Suemitsu, E. Shigeto, I. Yamadori, Y. Obayashi, H. Miyawaki, N. Dobashi, T. Matsushima, J. Takahara. (C)ERS Journals Ltd 1999.

ABSTRACT: The present study was designed to evaluate the pathological and immunohistochemical findings of Mycobacterium avium intracellulare complex (MAC) lung infection.

A retrospective study was performed in five cases with positive cultures for MAC in whom lung resections were performed between January 1989 and December 1996. A determination of whether or not MAC caused pulmonary disease was made using the 1997 criteria defined by the American Thoracic Society. In addition, MAC was cultured from all of the five lung specimens. Pathological and immunohistochemical findings as well as chest computed tomography (CT) findings were evaluated in these five patients.

Pathological findings of bronchiectasis, bronchiolitis, centrilobular lesion, consolidation, cavity wall and nodules were demonstrated, respectively, in relation to chest CT findings. Extensive granuloma formation throughout the airways was clearly demonstrated. Immunohistochemical staining demonstrated: 1) epithelioid cells and giant cells; 2) myofibroblasts extensively infiltrating the cavity wall; and 3) B-cells detected in aggregates in the vicinity of the epithelioid granulomas.

This study identified pathological and immunohistochemical characteristics of Mycobacterium avium complex infection relative to chest computed tomography findings and allowed the conclusion that bronchiectasis and bronchiolitis were definitely caused by Mycobacterium avium complex infection. Eur Respir J 1999; 13: 535-540.
*Kagawa Medical University, Kagawa, Japan. "Kochi Medical School, Kochi, Japan. **Mitoyo General Hospital, Mitoyo, Japan. National Hiroshima Hospital, Hiroshima, Japan ${ }^{+}$Okayama, University Medical School, Okayama, Japan. " Takamatsu Ritsurin Hospital, Takamatsu, Japan. \$. Kawasaki Medical School, Kawasaki, Japan.

Correspondence: J. Fujita, First Dept of Internal Medicine, Kagawa Medical School, 1750-1, Miki-cho, Kita-gun, Kagawa, 761-0793, Japan. Fax: 0118187 8912147

Keywords: Chest computed tomography findings

epithelioid cells

Mycobacterium avium intracellulare

complex infection

myofibroblasts

pathological and immunohistochemical

findings

surgical treatment

Received: June 181998

Accepted after revision October 251998
Pulmonary disease caused by Mycobacterium avium intracellulare complex (MAC) in patients without predisposing conditions has become an increasingly common clinical problem [1-5]. Although Moore [6] reported some pathological findings of atypical mycobacterium, there are few reports comparing pathological findings and radiological findings in patients with MAC infection. In addition, there are no reports which evaluate the type and distribution of inflammatory cells in pulmonary MAC infection. Furthermore, it has been argued whether bronchiectasis is truly caused by MAC infection or is the predisposing condition favouring MAC colonization [6$10]$. Based on this background, this study was designed to evaluate the pathological and radiological findings in the lungs of patients undergoing lung surgery for MAC infection and to evaluate immunohistochemically type and distribution of infiltrating cells.

For editorial comments see page 479
Materials and methods

Patients

A retrospective study was performed in five cases with MAC-positive sputum in whom surgical resection of the lung was performed between January 1989 and December 1996. The criteria for defining nontuberculous mycobacteria (NTM) pulmonary disease were those of the American Thoracic Society (1997) [5]. In addition, MAC was cultured from resected lung specimens in all patients. Characteristics of these five patients are listed in table 1 . No patient had acquired immune deficiency syndrome (AIDS) or any underlying lung disease. In four of the five patients, there was a history of dust exposure.

\section{Radiological evaluation}

Chest computed tomography (CT) (standard CT) was performed in all five patients and chest $\mathrm{CT}$ findings were 
Table 1. - Patient characteristics

\begin{tabular}{|c|c|c|c|c|c|c|}
\hline Case No. & Age* yrs & Sex & Occupation & Date of operation & Operation & Smear \\
\hline 1 & 56 & $\mathrm{M}$ & Steel industry worker & $16 / 2 / 89$ & Right upper lobectomy & Positive \\
\hline 2 & 33 & M & Welder & $14 / 10 / 91$ & $\begin{array}{l}\text { Partial resection of the cavity in the } \\
\text { anterior segment of LUL }\end{array}$ & Negative \\
\hline 3 & 72 & M & Carpenter & $28 / 9 / 92$ & $\begin{array}{c}\text { Partial resection of posterior segment } \\
\text { of RUL }\end{array}$ & Positive \\
\hline 4 & 42 & $\mathrm{~F}$ & Housewife & $25 / 7 / 96$ & Right upper lobectomy & Positive \\
\hline 5 & 48 & M & Steel industry worker & 29/7/96 & Left upper lobectomy & Positive \\
\hline
\end{tabular}

M: male; F: female; RUL: right upper lobectomy; LUL: left upper lobectomy. *: at the time of operation.

analysed by three radiologists. Chest CT findings were classified as: 1) bronchiectasis; 2) cavity formation; 3 ) centrilobular nodules (defined as increased attenuation in the area around the terminal or respiratory bronchioles, multiple, well-circumscribed, with the majority of the nodules around $5 \mathrm{~mm}$ ); 4) consolidation; and 5) nodules (>10 mm). Bronchiectasis was diagnosed when the diameter of the bronchi was larger than that of the accompanying vessels [6].

\section{Pathological and immunohistochemical findings}

Resected lung specimens were stained with haematoxylin and eosin. To evaluate the types of inflammatory cells, immunohistochemical staining was performed, employing the avidin-biotin peroxidase complex method (Dako LSAB kit-peroxidase; DAKO Corp., Kyoto, Japan) using a panel of monoclonal antibodies (mAbs) identifying specific inflammatory cell types. The mAbs used were: antiCD68 (1:100) to distinguish monocytes and macrophages (DAKO Corp., 051-M814), anti-UCHL-1 (1:30) to distinguish T-cells (DAKO Corp., 076-M0742), anti-L26 (1:50) to distinguish B-cells (DAKO Corp., 091-M755), anti- $\alpha$ smooth muscle actin $(1: 200)$ to distinguish myofibroblasts (DAKO Corp., 093-M851), anti-KL-6 $\left(10 \mathrm{mg} \cdot \mathrm{mL}^{-1}\right)$ to distinguish epithelial cells (a gift from N. Kohno, Second Department of Internal Medicine, Ehime University School of Medicine, Ehime, Japan), and the polyclonal antibody to S-100 protein (1:800) to distinguish Langerhans and/or dendritic cells (DAKO Corp., 129-Z0311). In order to in- crease the immunoreactivity, preincubation with $0.1 \%$ pronase at $37^{\circ} \mathrm{C}$ for $20 \mathrm{~min}$ was performed before the CD68 antibody staining.

\section{Results}

Table 2 shows the preoperative chest CT findings and pathological findings in the resected lung of the five patients. Centrilobular nodules, bronchiectasis, bronchiolitis and cavity formation were observed in all patients. Nodules $>10 \mathrm{~mm}$ and consolidation were observed in two patients.

The preoperative chest CT findings of bronchiectasis and bronchiolitis are demonstrated in figure 1. Radiologically, the thickening of the walls of large to small airways was clearly demonstrated. Pathologically, extensive peribronchial infiltration of mononuclear cells as well as epithelioid cells, surrounding bronchi (fig. 2a) as well as bronchioles (fig. 2b) was clearly shown. Ulceration of the bronchial wall was frequently observed associated with the derangement or the discontinuity of the muscle layer, as revealed by anti- $\alpha$-smooth muscle actin staining. In addition, narrowing of bronchioles was frequently observed. In some areas, necrotic material was released into the lumen of the bronchiole. Peribronchial granulomas were observed protruding into the bronchial lumen, resulting in the marked narrowing of the bronchiole (fig. 3).

The preoperative chest CT findings of centrilobular nodules (defined as increased attenuation in the area around

Table 2. - Correlations between preoperative chest computed tomography (CT) findings and pathological findings of the resected lung

\begin{tabular}{|c|c|c|c|c|c|c|}
\hline Case & Centrilobular & Bronchiectasis & Cavity & Nodules & Bronchiolitis & Consolidation \\
\hline \multicolumn{7}{|c|}{ Preoperative chest CT findings* } \\
\hline 1 & Yes & Yes & Yes & No & Yes & Yes \\
\hline 2 & Yes & Yes & Yes & Yes & Yes & No \\
\hline 3 & Yes & Yes & Yes & No & Yes & No \\
\hline 4 & Yes & Yes & Yes & No & Yes & No \\
\hline 5 & Yes & Yes & Yes & Yes & Yes & Yes \\
\hline \multicolumn{7}{|c|}{ Pathological findings of the resected lung ${ }^{+}$} \\
\hline 1 & Yes & Yes & Yes & No & Yes & Yes \\
\hline 2 & NE & $\mathrm{NE}^{+}$ & Yes & $\mathrm{NE}$ & $\mathrm{NE}$ & $\mathrm{NE}$ \\
\hline 3 & Yes & Yes & Yes & No & Yes & No \\
\hline 4 & Yes & Yes & Yes & No & Yes & No \\
\hline 5 & Yes & Yes & Yes & Yes & Yes & Yes \\
\hline
\end{tabular}

NE: not evaluable. ${ }^{*}$ : chest $\mathrm{CT}$ readings were examined by a radiologist blinded to the pathological reports; ${ }^{+}$: pathological findings were evaluated by two pathologists blinded to the chest CT readings; ${ }^{\star}$ : NE since only a cavity lesion was resected. 


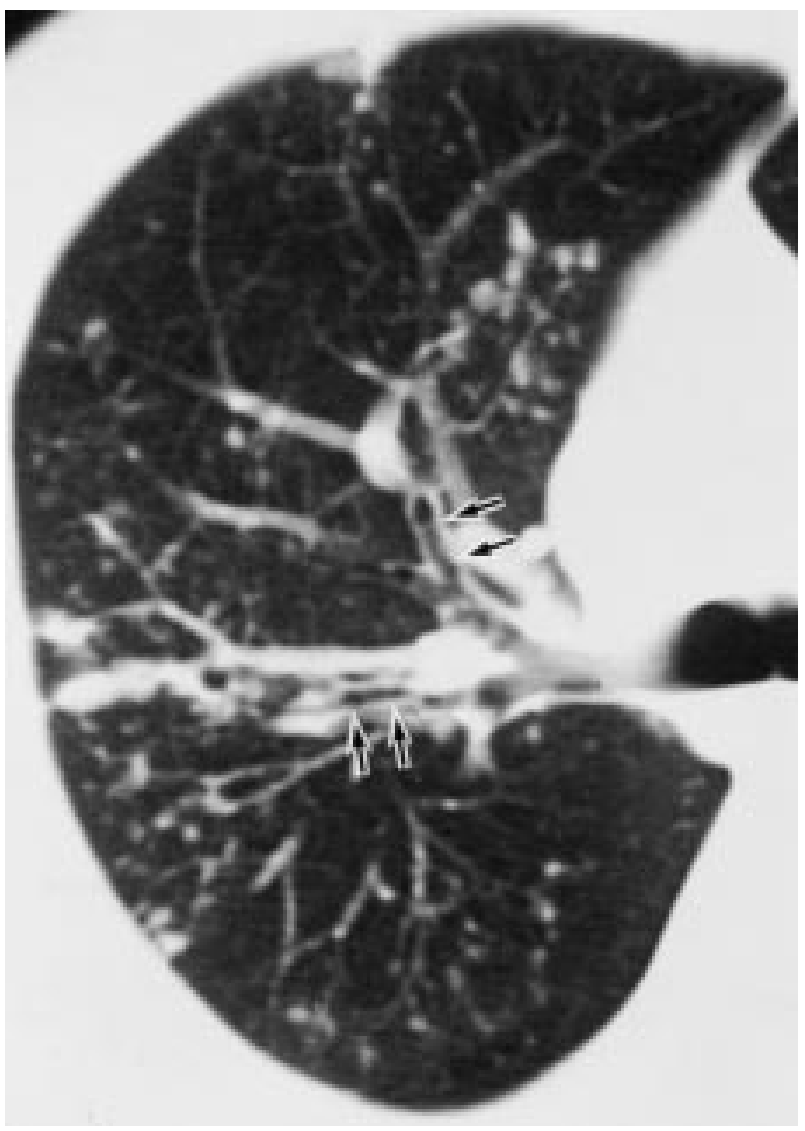

Fig. 1. - Preoperative chest computed tomography findings of bronchiectasis and bronchiolitis. Thickening of the bronchial walls from large to small airways were clearly demonstrated (arrows).

the terminal or respiratory bronchioles, multiple, well-circumscribed, and the majority of nodules $\sim 5 \mathrm{~mm}$ ) and nodules $>10 \mathrm{~mm}$ are demonstrated in figure 4. Haematoxylin and eosin staining of these centrilobular nodules showed 2-3 mm discrete granulomas which consisted of mononuclear cells and epithelioid cells (fig. 5). There were also adjacent areas of inflammatory infiltration of the alveolar walls. The pathological examination of nodules $>10 \mathrm{~mm}$ in diameter demonstrated caseous necrosis in the centre of the granulomas. Immunohistochemical staining for bronchial epithelial cells demonstrated that granuloma formation occurred in the alveolar walls and caused the collapse or the enfolding of several alveolar spaces within the granulomatous lesion. Immunohistochemical evaluation of the cavity wall demonstrated that epithelioid cells were arranged in the peripheral part of the caseous necrosis (fig. 6), and myofibroblasts were extensively found in the outer circumference of the epithelioid cell layer (fig. 7).

Preoperative chest CT findings of consolidation are shown in figure 8 . These specimens showed areas of inflammatory thickening of the alveolar walls, loosely grouped granulomas, and/or coalescent inflammatory infiltrates completely replacing the normal alveoli (fig. 9). Oedema in the alveoli adjacent to granulomas was also frequently observed. In some areas, a focal desquamative interstitial pneumonia-like pattern was observed (data not shown).
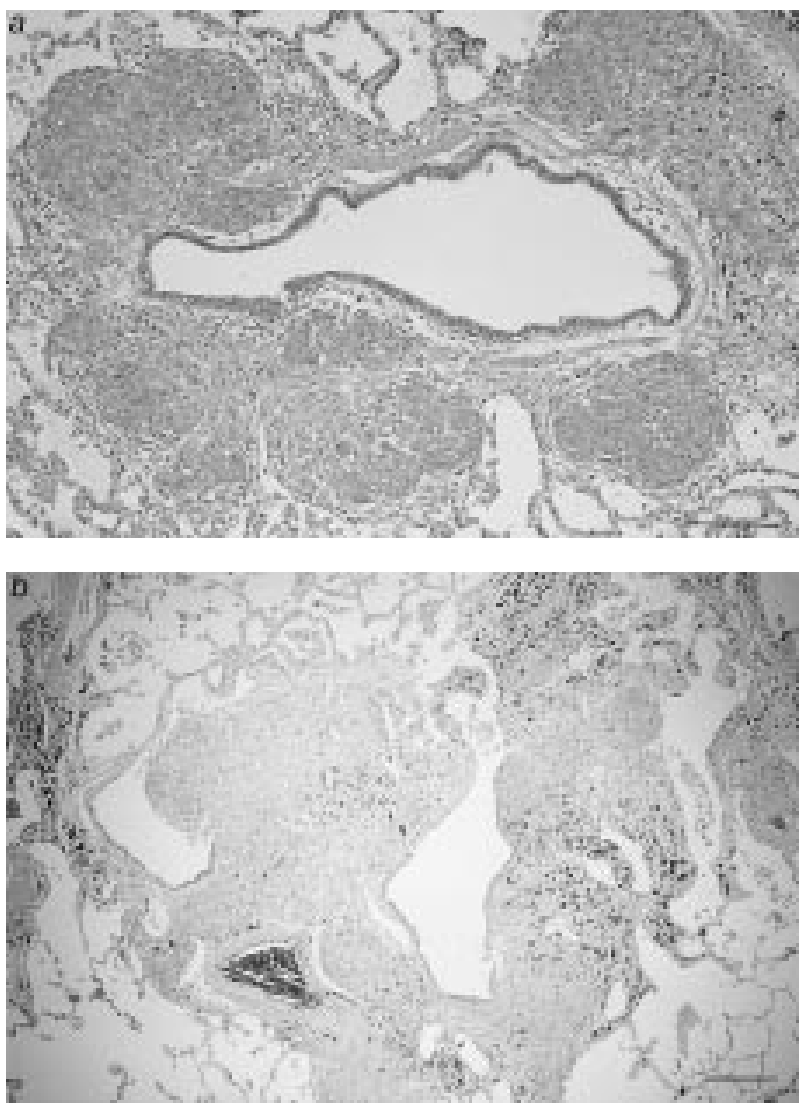

Fig. 2. - Haematoxylin and eosin staining of bronchiectasis and bronchiolitis. extensive peribronchial infiltration of mononuclear cells as well as epithelioid cells, surrounding a) a bronchus or b) a bronchiole, are demonstrated. (Internal scale bar $\mathrm{a}=800 \mu \mathrm{m}, \mathrm{b}=1.5 \mathrm{~mm}$.)

Immunohistochemical evaluation of the cells in the granuloma demonstrated epithelioid cells and multinucleated giant cells. In addition, aggregates of B-cells were frequently observed in the vicinity of the granuloma. In contrast, T-cells were dispersed in the granuloma as well as in the surrounding tissue. There were few dendritic cells in the granuloma. The immunohistochemical findings on resected lungs are summarized in table 3.

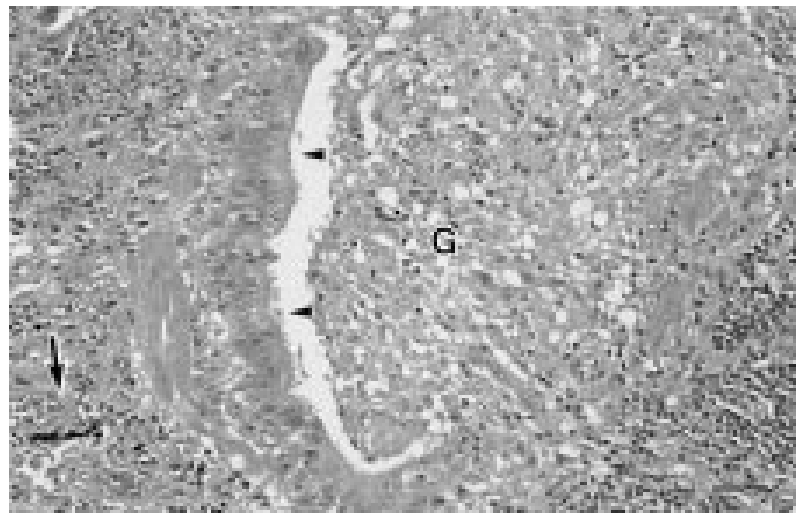

Fig. 3. - Narrowing of bronchiolar air space by a granuloma (G) protruding into the bronchial lumen. Note the covering ciliated epithelial cells (arrowheads). Anthracotic deposits can be observed around this small airway (arrow). (Internal scale bar=50 $\mu \mathrm{m}$.). 


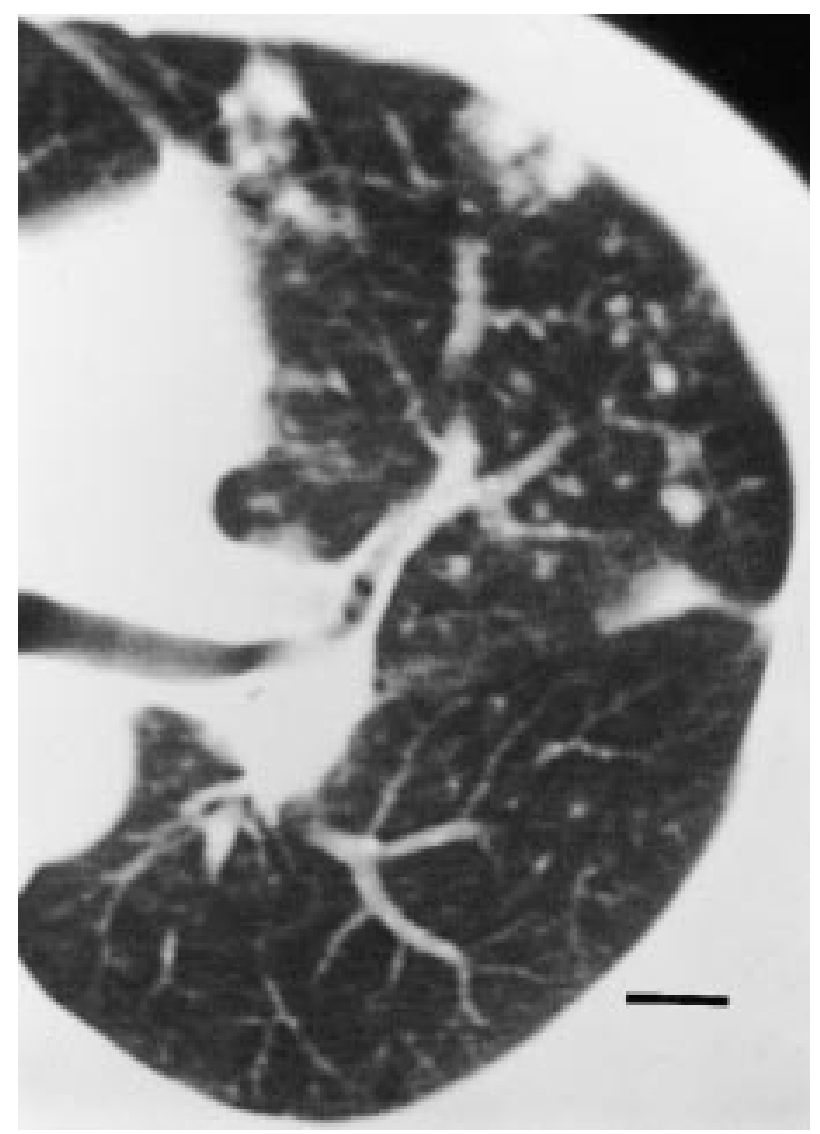

Fig. 4. - Preoperative chest computed tomography findings of centrilobular nodules and nodules $>10 \mathrm{~mm}$. (Internal scale bar $=1 \mathrm{~cm}$.)

MAC were stained only in the caseating areas of the granuloma. Staining to MAC was positive in four out of the five cases.

\section{Discussion}

In the present study, the pathological and radiological findings associated with MAC infection in resected lungs was demonstrated. Extensive granuloma formation caused by MAC infection was observed throughout the lung.

MAC is a ubiquitous pathogen that has been found in soil, domestic tap water and animals [11]. Pulmonary disease caused by MAC infection has a wide spectrum of clinical presentations from simple pneumonic infiltrate to progressive destructive disease [11-13]. Many authors believed that in the majority of patients with MAC pulmonary disease there were chronic underlying lung diseases such as pneumoconiosis, bronchiectasis, chronic bronchitis and emphysema [1, 11]. More recently, however, MAC has increasingly been recognized as a primary infectious agent in immunocompetent patients, especially in females without pre-existing pulmonary disease [1]. Although exposure to MAC often occurs without any clinical manifestation, clinical manifestations may range from no symptoms to signs of destructive or even fatal disease [11]. Interestingly, in the present study, four of the five patients were male and had a history of dust exposure and, consistent with exposure, anthracotic deposits were observed around small airways in the resected lungs. a)

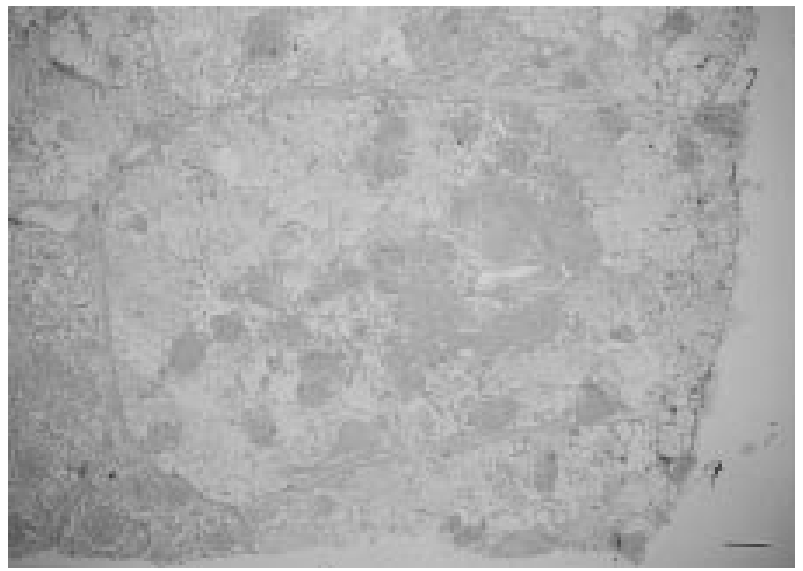

b)

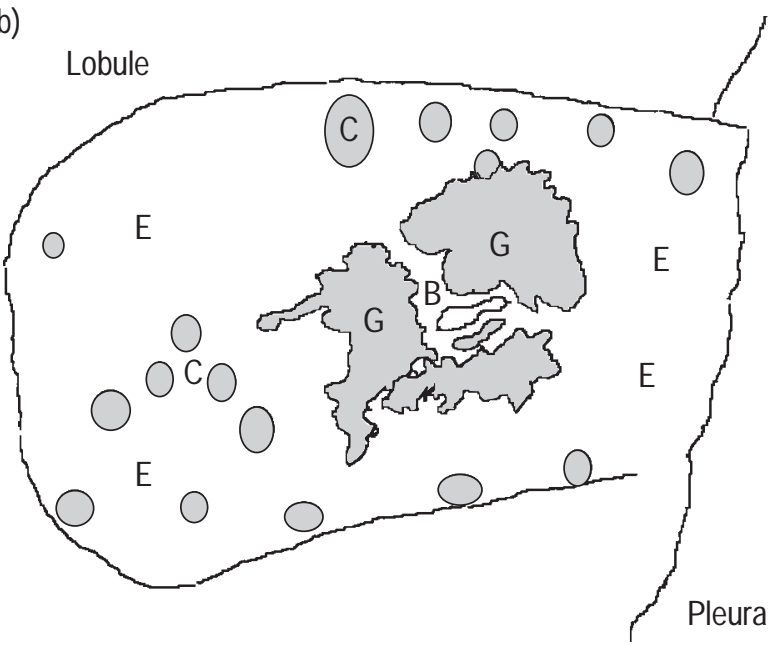

Fig. 5. - a) Haematoxylin and eosin staining of centrilobular nodules in the lobule. b) Diagrammatic representation of (a) showing peribronchial granuloma (G), bronchiole (B), oedema (E) and centrilobular nodules $(\mathrm{C})$. (Internal scale bar=3 $\mathrm{mm}$.)

A number of reports on CT evaluation of patients with MAC have recently been published [6-10]. It has been reported that bronchiectasis with discrete nodules are an important radiological finding of MAC [6-10]. HARTMAN et al. [7] suggested that the concomitant findings of

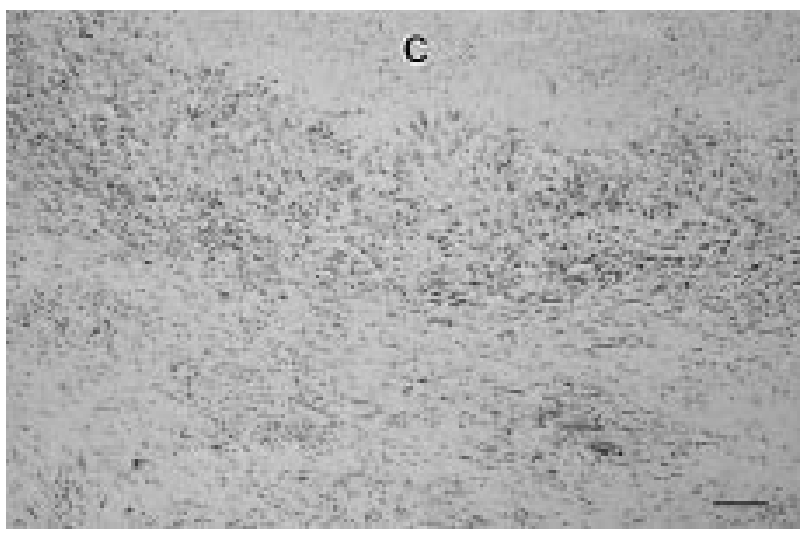

Fig. 6. - Immunohistochemical staining of epithelioid cells in the cavity wall by the anti-CD68 monoclonal antobody. C: the site of caseous necrosis. (Internal scale bar=100 $\mu \mathrm{m}$.). 


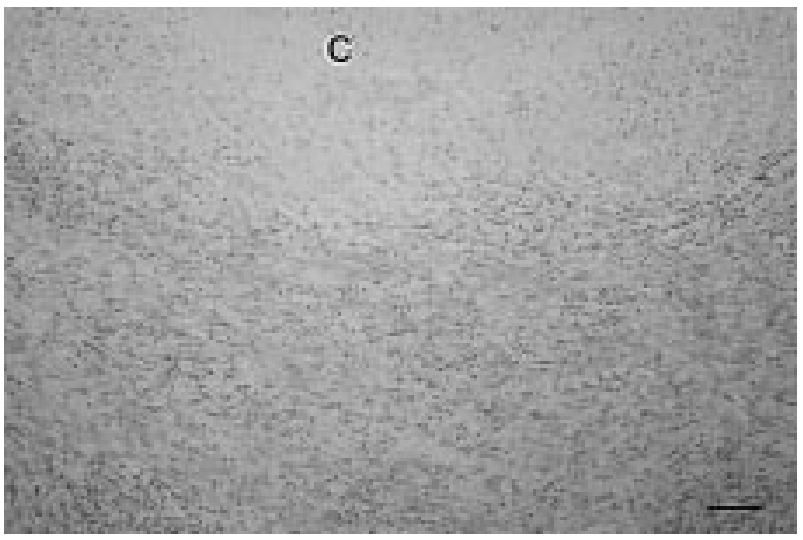

Fig. 7. - Immunohistochemical staining of myofibroblasts in the cavity wall by the anti- $\alpha$-smooth monoclonal antibody. C: the site of caseous necrosis. (Internal scale bar=100 $\mu \mathrm{m}$.)

bronchiectasis and multiple small well-circumscribed lung nodules are indicative of infection or colonization with MAC. They identified a subtype of patients (predominantly older females with no underlying malignancy or clinically evident immunodeficiency) with MAC who presented with this characteristic pattern on chest CT scans.

MOORE [6] demonstrated centilobular nodules, bronchiectasis and nodules in a few patients, but no cavitation or consolidation. In the present study, the characteristic pathological finding of pulmonary MAC disease was extensive

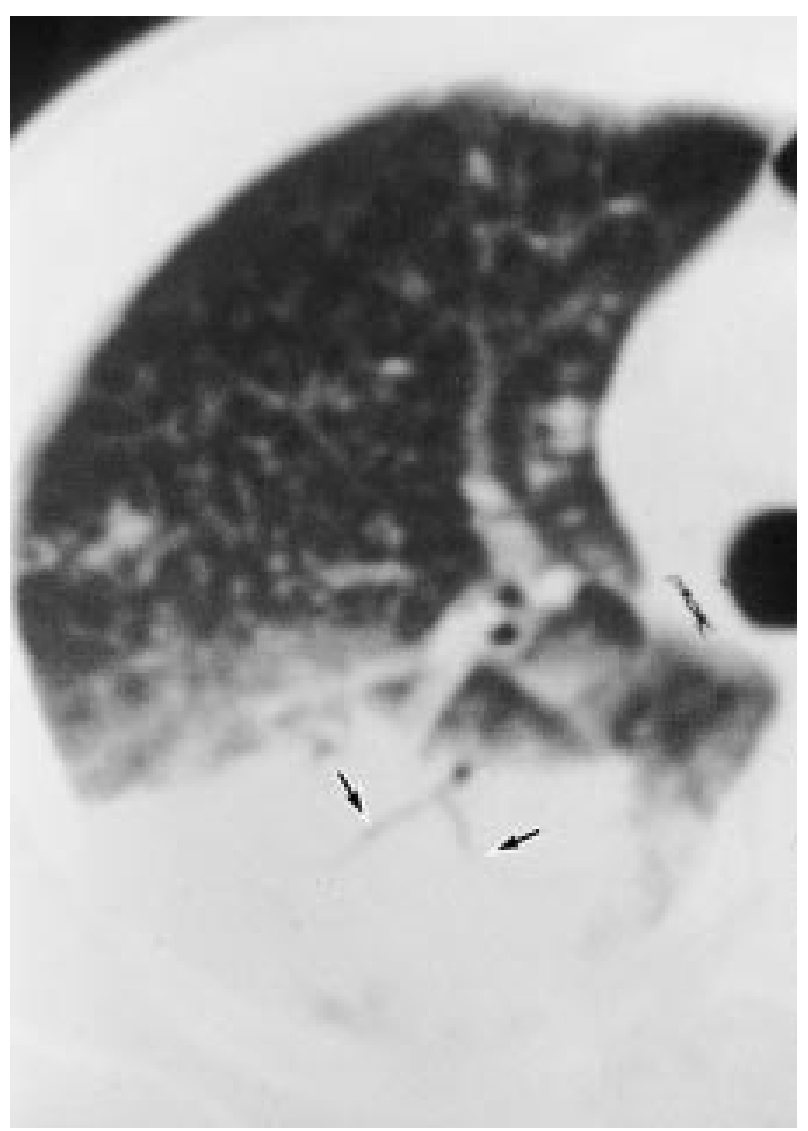

Fig. 8. - The pre-operative computed tomography findings of consolidation. Air-space consolidation and air bronchogram (arrows) are shown.

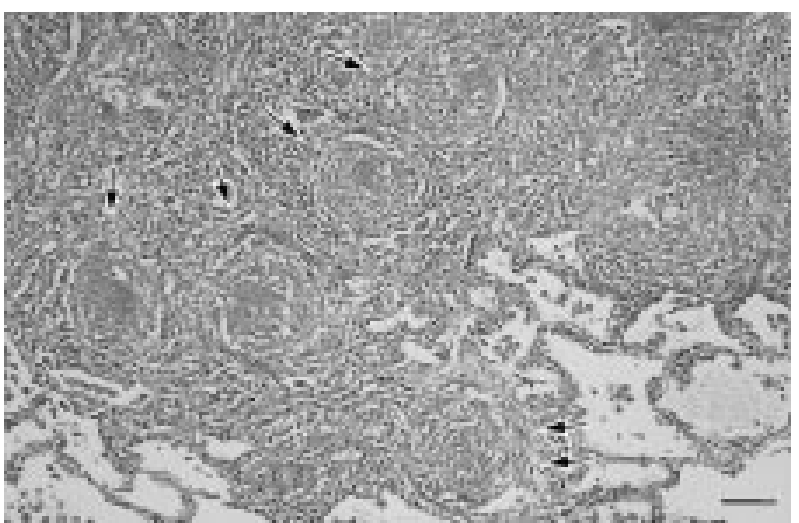

Fig. 9. - Haematoxylin and eosin staining of consolidation. Arrows indicate loosely grouped epithelioid cell granuloma. These granulomas are replacing normal alveoli (double arrow) in association with collapses and enfolding of the alveolar spaces. Inflammatory thickening of the alveolar walls is also seen. (Internal scale bar=100 $\mu \mathrm{m}$.).

granuloma formation throughout the airways. Peribronchial granuloma formation was observed from the large airway to the bronchiole. In the bronchiole, a peribronchial granuloma protruded into the lumen which resulted in the narrowing of the bronchiole. In addition, ulceration of the bronchial wall with ensuing disruption of the muscle layer was frequently observed, which may have caused bronchiectatic changes. In some areas, necrotic materials were found in the lumen of the bronchiole, suggesting that centrilobular nodules might have been caused by transbronchial dissemination of this necrotic material.

In the air-spaces, loosely grouped granulomas and/or coalescent inflammatory infiltrates completely replaced normal alveoli. Oedema and congestion in alveolar lesions adjacent to the granulomas were frequently observed, suggesting that local circulation in the microvessels and lymphatics were also disturbed by MAC infection. Collapse or enfolding of several alveolar spaces together with focal desquamative interstitial pneumonia may have been partially responsible for these air-space lesions.

In addition, this study identified the major types of inflammatory cells and their distribution in the lesions. In the cavity wall, myofibroblasts were shown to infiltrate the outer wall of epithelioid cells. As it has been reported that transforming growth factor (TGF)- $\beta$ induces myofibroblasts, TGF- $\beta$ released from the epithelioid cells may have been responsible for the appearance of myofibroblasts [14]. Interestingly, the distribution patterns of T-cells and B-cells were different. Aggregates of B-cells were frequently observed surrounding the granulomas. In contrast, T-cells were dispersed in and around the granulomas.

In conclusion, although future studies evaluating the differences in pathological and immunohistochemical findings between Mycobacterium avium intracellulare complex infection and Mycobacterium tuberculosis infection are necessary, this study characterizes the typical lesions of Mycobacterium avium intracellulare complex pulmonary infection. The extensive peribronchial granuloma formation along the airways, and also in areas without bronchiectasis and ulcerations of the bronchial wall let us assume that bronchiectasis and bronchiolitis is definitely caused by Mycobacterium avium intracellulare complex infection. 
Table 3. - Immunohistochemical findings of the resected lung

\begin{tabular}{|c|c|c|}
\hline Antibodies used & Stained cells & Findings \\
\hline Anti-CD68 & Monocytes and macrophages & $\begin{array}{l}\text { Epithelioid cells and multinucleated giant cells in granulomas were stained, } \\
\text { together with alveolar macrophages }\end{array}$ \\
\hline Anti-UCHL-1 & pan-T cells & Dispersed T-cells were observed in the granuloma as well as surrounding parts \\
\hline Anti-L26 & pan-B cells & Aggregate B-cells were observed in the vicinity of the granuloma \\
\hline $\begin{array}{l}\text { Anti- } \alpha \text {-smooth } \\
\text { muscle actin }\end{array}$ & Myofibroblasts & $\begin{array}{l}\text { Myofibroblasts were extensively detected along the cavity wall in the outer } \\
\text { circumference of the epithelioid cells }\end{array}$ \\
\hline Anti-KL-6 & Pulmonary epithelial cells & Collapsed or enfolded alveolar spaces within the granuloma \\
\hline Anti-S-100 & Dendritic cells & Few dendritic cells in the granuloma \\
\hline
\end{tabular}

\section{References}

1. Prince DS, Peterson DD, Steiner RM, et al. Infection with Mycobacterium avium complex in patients without predisposing conditions. N Engl J Med 1989; 321: 863-868.

2. O'Brien RJ, Geiter W, Snider DE Jr. The epidemiology of nontuberculous mycobacterial diseases in the United States: results from a national survey. Am Rev Respir Dis 1987; 135: 1007-1014

3. Tanaka E, Amitani R, Niimi A, Suzuki K, Murayama T, Kuze F. Yield of computed tomography and bronchoscopy for the diagnosis of Mycobacterium avium complex pulmonary disease. Am J Respir Crit Care Med 1997; 155: 2041-2046.

4. Wallace RJ, O'Brien R, Glassroth J, Raleigh J, Dutt A. Diagnosis and treatment of disease caused by nontuberculous mycobacteria. Am Rev Respir Dis 1990; 142: 940 953.

5. Wallace RJ, Glassroth J, Griffith DE, Olivier KN, Cook JL, Gordin F. American Thoracic Society: diagnosis and treatment of disease caused by nontuberculous mycobacteria. Am J Respir Crit Care Med 1997; 156: S1-S25.

6. Moore EH. Atypical mycobacterial infection in the lung: CT appearance. Radiology 1993; 187: 777-782.

7. Hartman TE, Swensen SJ, Williams DE. Mycobacterium avium-intracellulare complex: evaluation with CT. Radiology 1993; 187: 23-26.

8. Swensen SJ, Hartman TE, Williams DE. Computed tomo- graphic diagnosis of Mycobacterium avium-intracellulare complex in patients with bronchiectasis. Chest 1994; 105 : 49-52.

9. Primack SL, Logan PM, Hartman TE, Lee KS, Muller NL. Pulmonary tuberculosis and Mycobacterium aviumintracellulare: a comparison of CT findings. Radiology 1995; 194: 413-417.

10. Lynch DA, Simone PM, Fox MA, Bucher BL, Heinig MJ. CT features of pulmonary Mycobacterium avium complex infection. J Comput Assist Tomogr 1995; 19: 353-360.

11. Jarad NA, Demertzis P, Meecham Jones DJ, et al. Comparison of characteristics of patients and treatment outcome for pulmonary non-tuberculous mycobacterial infection and pulmonary tuberculosis. Thorax 1996; 51: 137-139.

12. Guthertz LS, Damsker B, Bottone EJ, Ford EG, Midura TF, Janda JM. Mycobacterium avium and Mycobacterium intracellulare infections in patients with and without AIDS. J Infect Dis 1989; 160: 1037-1041.

13. Ahkee S, Srinath L, Huang AK, Ramirez JA. Clinical significance of mycobacterium other than tuberculosis isolated from respiratory specimens at University Hospital. J Ky Med Assoc 1995; 93: 53-55.

14. Desmouliere A, Geinoz A, Gabbiani F, Gabbiani G. Transforming growth factor- $\beta 1$ induces $\alpha$-smooth muscle actin expression in granulation tissue myofibroblasts and in quiescent and growing cultured fibroblast. J Cell Biol 1993; 22: 103-111. 